\title{
Plasma Electrode Pockels Cell for the National Ignition Facility
}

\author{
M. A. Rhodes \\ S. Fochs \\ P. Biltoft \\ T. Alger \\ B. Funkhouser \\ C. D. Boley
}

This paper was prepared for submittal to the Third Annual International Conference on Solid State Lasers for Application (SSLA) to Inertial Confinement Fusion (ICF) Monterey, California

June $7-12,1998$

July 28,1998

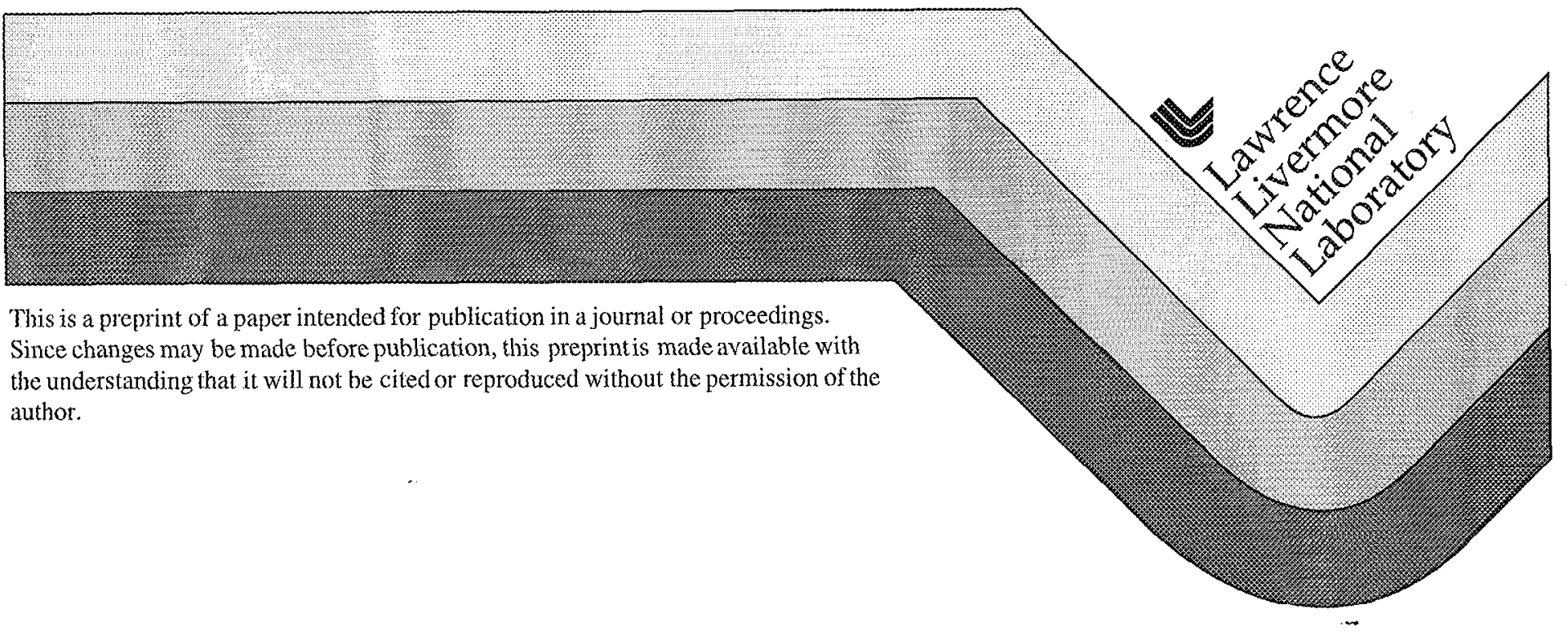




\section{DISCLAIMER}

This document was prepared as an account of work sponsored by an agency of the United States Government. Neither the United States Government nor the University of California nor any of their employees, makes any warranty, express or implied, or assumes any legal liability or responsibility for the accuracy, completeness, or usefulness of any information, apparatus, product, or process disclosed, or represents that its use would not infringe privately owned rights. Reference herein to any specific commercial product, process, or service by trade name, trademark, manufacturer, or otherwise, does not necessarily constitute or imply its endorsement, recommendation, or favoring by the United States Government or the University of California. The views and opinions of authors expressed herein do not necessarily state or reflect those of the United States Government or the University of California, and shall not be used for advertising or product endorsement purposes. 
Plasma Electrode Pockels Cell for the National Ignition Facility

Mark A. Rhodes, Scott Fochs, Peter Biltoft Terry Alger, Bill Funkhouser, C.D. Boley

Lawrence Livermore National Laboratory, Livermore, CA 94550

\begin{abstract}
The National Ignition Facility (NIF), now under construction at Lawrence Livermore National Laboratory, will be the largest laser fusion facility ever built. The NIF laser architecture is based on a multi-pass power amplifier to reduce cost and maximize performance. A key component in this laser design is an optical switch that closes to trap the optical pulse in the cavity for four gain passes and then opens to divert the optical pulse out of the amplifier cavity. The switch is comprised of a Pockels cell and a polarizer and is unique because it handles a beam that is $40 \mathrm{~cm} \times 40 \mathrm{~cm}$ square and allows close horizontal and vertical beam spacing. Conventional Pockels cells do not scale to such large apertures or the square shape required for close packing. Our switch is based on a Plasma-Electrode Pockels Cell (PEPC).
\end{abstract}

In a PEPC, low-pressure helium discharges $(1-2 \mathrm{kA})$ are formed on both sides of a thin slab of electro-optic material. Typically, we use $\mathrm{KH}_{2} \mathrm{PO}_{4}$ crystals (KDP). The discharges form highly conductive, transparent sheets that allow uniform application of a high-voltage pulse $(17 \mathrm{kV})$ across the crystal. A $37 \mathrm{~cm} \times 37 \mathrm{~cm}$ PEPC has been in routine operation for two years on the $6 \mathrm{~kJ}$ Beamlet laser at LLNL. For the NIF, a module four apertures high by one wide $(4 \times 1)$ is required. However, this $4 \times 1$ mechanical module will be comprised electrically of a pair of $2 \times 1$ sub-modules.

Last year (FY 97), we demonstrated full operation of a prototype $2 \times 1$ FEPC. In this PEPC, the plasma spans two KDP crystals. A major advance in the $2 \times 1$ PEPC over the Beamlet PEPC is the use of anodized aluminum construction that still provides sufficient insulation to allow formation of the planar plasmas. In this paper, we discuss full $4 \times 1$ NIF prototypes.

Keywords: Plasma, Pockels Cell, Laser Fusion, High-Voltage

\title{
2. INTRODUCTION
}

In this paper, we discuss our efforts to develop a plasma-electrode Pockels cell (PEPC) to be used as part of an optical switch in the laser portion of the National Ignition Facility. Previously, we proved that the PEPC is a viable technology in high-energy pulsed lasers by fielding a $37 \times 37 \mathrm{~cm}^{2}$ PEPC on the Beamlet laser. ${ }^{1}$

While the Beamlet PEPC has proven to be an effective and reliable part of the laser, its design will not scale to the requirements of the NIF laser. For NIF, we must arrange 192 PEPC apertures in vertical columns four apertures high. It is also required that the PEPC apertures line up with the cavity amplifier apertures. This leaves little space for structural material between the PEPC apertures and would not be possible with the polyethylene construction used for the Beamlet PEPC. Starting in FY96, we began developing a new PEPC construction based on anodized aluminum. We envisioned a $4 \times 1$ PEPC line replaceable unit (LRU) comprised electrically of a pair of $2 \times 1$ PEPC units. Compared to the Beamlet PEPC, the plasma would now have to span two full apertures and the switch-pulse generators would have to apply voltage to a pair of KDP crystals in parallel. Also, because of the close horizontal packing of PEPC LRUs on NIF, we had to arrange all electrical and vacuum interfacing at the top and bottom ends. We proved that we could achieve all these design requirements in FY 97 by demonstrating operation of a twoaperture or $2 \times 1$ PEPC. This device is shown in Figure 1. Its performance results exceeded NIF requirements and are reported

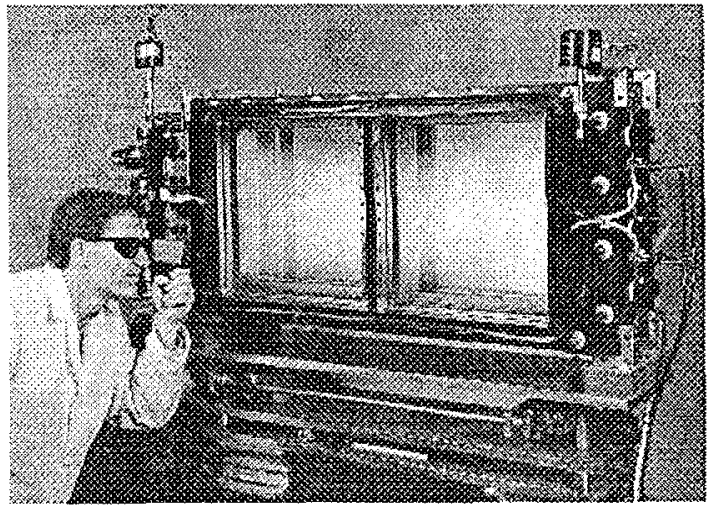

Figure 1. This two aperture, aluminum housing PEPC proved most of the design features required for a NIF PEPC.

elsewhere. ${ }^{2}$ For the full NIF $4 \times 1$ design we decided to split the prototyping efforts into two parallel efforts. In this last year 
we have constructed a "mechanical prototype" and an "operational prototype". Before presenting details of these prototypes, in Section II we present an overall description of the PEPC LRU design. In Section III, we will discuss the mechanical prototype. In Section IV we will present results from the operational prototype.

\section{PEPC LRU DESIGN}

In this section, we describe the overall design of the PEPC LRU. The operational part of the LRU is comprised of midplane sandwiched between a pair of housings. All other components attach to the housings. The midplane is a sheet of glass with four apertures cut out. In each of these apertures, we have potted a $40 \mathrm{~cm} \times 40 \mathrm{~cm} \times l \mathrm{~cm}$ plate of KDP crystal. The potting material is a high-grade silicone rubber. The housings are made of aluminum with a hard anodize coating. This coating provides insulation so that the conductive housing does not short the plasma current. Other components include windows, window retainers, anodes, cathodes, vacuum baffles, and vacuum plumbing. The windows are fused silica and rest on o-rings. During operation, the windows are held in place by atmospheric pressure since the interior is under vacuum. However, the windows are also held in place by retainer rings for times when the vacuum system is off.

Cathodes are the negative electrodes for the plasma discharge. There is one cathode for each of the four plasma quadrants. We use a planar magnetron catbode design. High-field permanent magnets confine plasma near the cathode surface. This lowers the required operating pressure and provides good discharge uniformity. The cathodes have a graphite cover. Since sputtering of the cathode is inevitable, we must control deposition of the sputtered material on the optical surfaces. By using graphite, we assurc that the sputtered material is carbon. The operating gas is helium plus $1 \%$ oxygen. The oxygen reacts with the sputtered carbon to produce carbon monoxide and carbon dioxide. Since these are gaseous species, they are pumped away by the vacuum system.

Anodes are the positive electrodes for the plasma discharge. We segment the anode into six pieces per plasma discharge. We assure uniform plasma current to each anode segment with an appropriate ballast resistor. Anode segmentation improves the plasma uniformity.

The entire LRU is pumped by a single turbomolecular-drag pump and backed by a suitable roughing pump. The interior of the housings is pumped through holes in the cathodes. These holes lead to tubes that penetrate the housing ends. The pump out tubes connect to an insulated vacuum baffle structure. The baffle structure provides electrical isolation between the two housings when the $17 \mathrm{kV}$ switch-pulse is applied. Electrons from the plasma try to flow to the grounded vacuum structure during this pulse. The baffles prevent this while still allowing high vacuum conductance. The vacuum system provides a base pressure of around $5 \times 10^{-5}$ torr. The working gas is fed in with a feedback controlled mass flow controller. This gas system is set up to maintain constant pressure rather than constant flow. Our operating pressure is typically 65 mitorr.

The operational portion of the PEPC LRU, described above, mounts in an " $L$ " shaped frame. Also attached to this frame is a three point kinematic mounting system. The other side of these mounts will be attached to the NIF periscope structure. At the bottom of the frame is an interface plate. All interfaces (electrical, gas, cooling, vacuum, diagnostics, and controls) pass through this bottom plate.

\section{THE MECHANICAL PROTOTYPE}

The mechanical prototype, shown in Figure 2, allows us to test the mechanical aspects of the LRU including kinematic repeatability, vibration testing, LRU handling, and interfaces. The mechanical prototype uses a pair of real PEPC housings but uses a "dummy" midplane (no KDP) and "dummy"

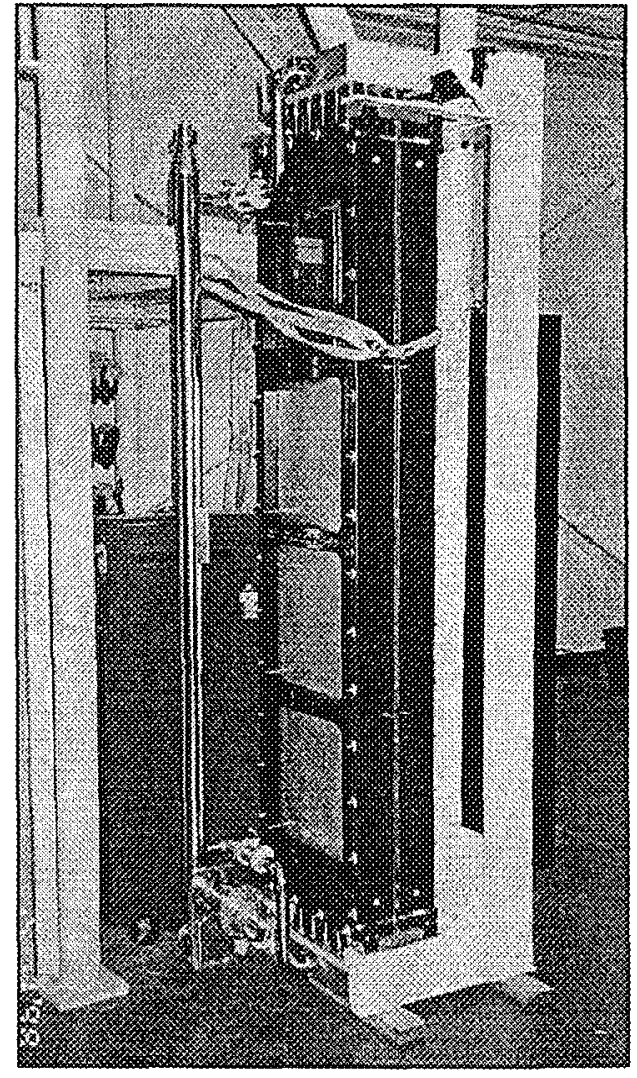

Figure 2. Mechanical prototype of the NIF PEPC LRU. 
windows (aluminum and acrylic). The frame is the actual NIF design as are the kinematic mounts. A vacuum system is included but no gas systen (at this time).

To date we have performed mounting repeatability testing. The requirement is to place the LRU within $\pm 1 \mathrm{~mm}$. Our measured performance is that the LRU locates within $\pm 0.025 \mathrm{~mm}$, forty times better than required.

\section{THE OPERATIONAL PROTOTYPE}

The operational prototype, shown in Figure 3 , is designed to allow testing of the electro-optic operation of the $4 \times 1$ PEPC. In this prototype, we forego the external frame. The midplane contains four, full-size, rapid-growth ${ }^{3} \mathrm{KDP}$ crystals. The windows are fused silica made to NIF specifications. We have not anti-reflection (AR) coated the windows or crystal but we know from Beamlet experience that AR coatings do not affect PEPC operation. We evaluate the $4 \times 1$ PEPC with the apparatus shown schematically in Figure 4. The output coupler of a $10 \mathrm{~ns}$ pulsed $1.064 \mu \mathrm{m}$ laser is relay imaged to the plane of the KDP with a beam expanding telescope. The beam is shaped square and also passes through a polarizer to ensure that the PEPC is exposed to linearly polarized light. We use a system of beam splitters (not shown) to produce four identical full-aperture beams. After passing through the cell, the four beams are each relayed imaged to a CCD camera after passing through an analyzing polarizer. We determine PEPC performance by measuring extinction ratio (ER). We do this by comparing the CCD inage with and without the PEPC operating. The NIF requirements are that the average ER for each aperture must be greater than 100 while the worst spot can have an ER no lower than 50 . If the ER is 100 , this means that $99 \%$ of the light is in the proper polarization. An ER of 50 corresponds to $98 \%$ of the light in the proper polarization. Put another way, if the ER is 50 on some part of the aperture, a $2 \%$ amplitude modulation is introduced into the beam. Figure 5 shows the results. The average ER for each aperture is about 1200 (twelve times better than required) and the worst spots in

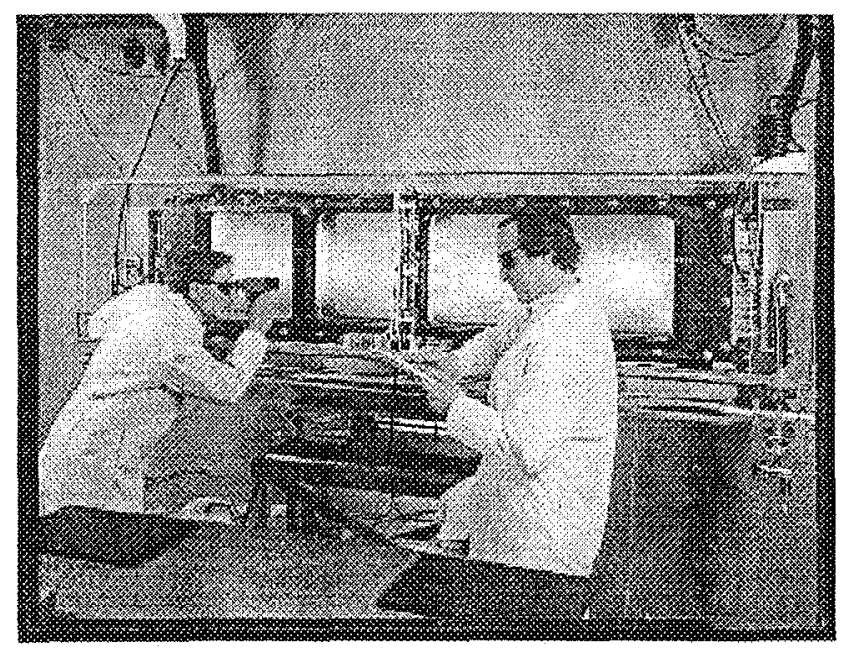

Figure 3 . We use this $4 \times 1$ operational prototype to test the optical switching performance of the NIF PEPC.

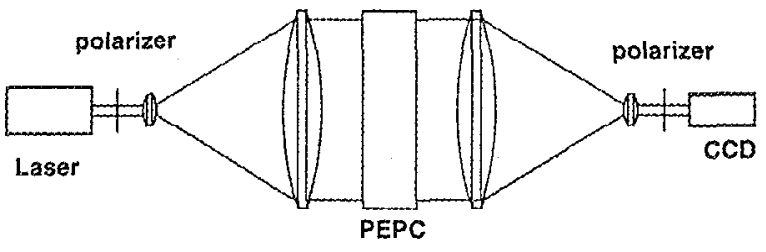

Extinction Ratio Imaging

Figure 4. Experimental apparatus used to measure optical switching performance of the NIF PEPC. each aperture are all better than 50 . Note that this data is highly reproducible. In the lab, we fire the PEPC system continuously every four seconds. When tuned to the right voltage, the data is the same within small deviations shot after shot. We have logged thousands of shots on this PEPC during the course of our experiments.

\section{CONCLUSION}

We have described the design of a four-aperture plasmaelectrode Pockels cell for use in the National Ignition Facility. The device is the active part of an optical switch that enables a multi-pass laser amplifier. We have proven the optical performance of our design by building and operating a full size operational prototype. We have separately proven the mechanical aspects of our design with a non-operating mechanical prototype. The kinematic mounting system locates the entire structure with a high degree of accuracy while the optical performance meets or exceeds the requirements by wide margins.

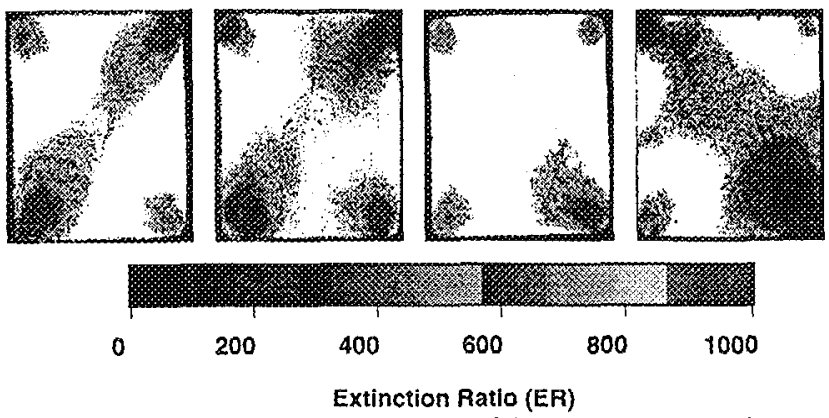

Figure 5. Extinction ratio images for each of the four apertures during normal PEPC operation. 


\section{ACKNOWLEDGMENTS}

This work was performed under the auspices of the US Department of Energy by Lawrence Livermore National Laboratory under the contract number W-7405-ENG-48.

\section{REFERENCES}

1. Mark A. Rhodes, B. Woods, J. J. DeYoreo, D. Roberts, L. J. Atherton, "Performance of larger-aperture optical switches for high-energy inertial confinement fusion lasers," Appl. Op. 34, 5312-5325 (1995).

2. Mark A. Rhodes, Scott Fochs, C. D. Boley, "Plasma Pockels Cell Based Optical Switch for the National Ignition Facility," International Conference on Plasma Science, San Diego, CA, 19-22 May (1997).

3. N. P. Zaitseva, J. J. DeYoreo, M. R. Dehaven, R. L. Vital, K. E. Montgomery, M Richardson., and L. J. Atherton, "Rapid growth of large-scale (40-55cm) $\mathrm{KH}_{2} \mathrm{PO}_{4}$ crystals," J. Cryst. Growth 180, 255 (1997). 